\title{
ANÁLISIS DEL CONTEXTO POLÍTICO, SOCIAL Y ECONÓMICO COMO BASE PARA LA FORMACIÓN DE PERSONAL TÉCNICO DE SALUD EN AMÉRICA LATINA
}

\author{
ANALYSIS OF THE POLITICAL, SOCIAL AND ECONOMIC CONTEXT AS A BASE FOR THE \\ PROFESSIONAL QUALIFICATION OF THE HEALTH TECHNICIAN IN LATIN AMERICA
}

Miguel Márquez ${ }^{1}$

Resumo Este artículo tiene como objetivo resaltar la importancia del análisis del contexto político, social y económico para conformar el marco conceptual que tiene como base el desarrollo de los programas de formación de personal en salud. El análisis se define en cuatro periodos. El primero, a finales del siglo XIX hasta principios del XX, se caracterizó por ser 'médico-céntrico'. En el segundo, se introdujeron los mecanismos de administración médica, el registro de datos y estadísticas, la práctica médica adquirió nuevas dimensiones lo cual posibilitó que se convirtiera en una práctica social y no individual. En el tercero, con el traslado de la organización hospitalaria de Europa hacia América y la incorporación de la tecnología moderna, el hospital pasó a ser parte de una organización médica y social, con la misión de proporcionar asistencia médico-sanitaria y el cuarto, sucedió en medio de una contradicción entre lo que se había avanzado en el desarrollo de los sistemas de salud, con el reconocimiento del derecho universal a la salud y la participación de la ciudadanía como sujeto y objeto de la atención, y la emergencia de la corriente globalizadora neoliberal, con la consideración del complejo proceso de atención de la salud como una mercancía.

Palavras-chave política de salud; formación profesional; trabajo.
Abstract The purpose of this article is to highlight the importance of the analysis of the political, social, and economic context to confirm the conceptual framework as the base for the development of the qualification programs for health professionals. The analysis is defined in four moments. The first, ever since the end of the 19th century through the early 20th century, was characterized by being 'physiciancentric'. The second, in which the medical administration and data and statistics registration were included, the medical practice gained new dimensions that allowed it to turn into a social, rather than an individual practice. In the third, with the transference of the hospital organization from Europe to America and the incorporation of modern technology, hospitals became part of a medical and social organization with the mission of allowing for medial and sanitary assistance. A fourth took place in the midst of a contradiction between what had progressed in the development of the health systems, with the acknowledgement of the universal rights to health and citizen participation as the subject and object of the care, and the appearance of the neoliberal globalizing trend, which considered the complex process of health care as merchandise.

Keywords health policy; professional qualification; work. 


\section{Antecedentes}

La formación del personal técnico de salud, a partir de la segunda mitad del siglo XIX, surgió unida a la práctica médica y su formación fue legitimada con la relación directa médico-auxiliar-paciente. Posteriormente, a fines del siglo XIX y comienzos del XX, se produjo la irrupción del conocimiento científico en física, química y matemáticas. Estos avances fueron aplicados a la práctica médica y, junto a la reforma hospitalaria en Europa (extendida rápidamente hacia América), permitieron la incorporación de recursos tecnológicos cada vez más sofisticados, como los rayos $\mathrm{x}$, los análisis de sangre, de orina, heces, del líquido cefalorraquídeo, los exudados bronquiales y las pruebas cutáneas. Todo ello demandó la emergencia de personal técnico más calificado, y determinó el nacimiento del personal técnico medio, que mantuvo la categoría de personal auxiliar como base para la atención directa al paciente. Con el avance en la aplicación de este modelo, centrado en la práctica hospitalaria, el hospital, con su estructura orgánica y funcional, pasó a constituir el marco de referencia para la formación del personal técnico de salud y, a partir de los años 50 del siglo XX, se llevó a las universidades y a sus facultades de medicina a ampliar su marco de referencia institucional y su trabajo directo con la comunidad, lo que permitió ampliar la visión del contexto de formación del personal profesional y técnico. En ese momento, también fueron incorporadas las dimensiones de promoción y prevención en salud y, por consiguiente, el contexto, lo cual generó planes y programas de formación que consideraron las variables políticas, sociales y económicas.

A partir de estos cambios, que surgieron después de la Segunda Guerra Mundial, se logró una mayor independencia en la estructura de los planes y programas a través de escuelas politécnicas de salud, de las cuales algunas en este siglo, se han convertido en universidades con igual nivel jerárquico y autonomía que las facultades de medicina.

A continuación, intentaremos profundizar en las modificaciones que se efectuaron en el análisis del campos político, social y económico, como soporte al marco conceptual en la elaboración de planes y programas para la formación de personal técnico de salud. De acuerdo con el análisis documental, podemos identificar cuatro etapas de este proceso: la primera, a fines del siglo XIX; la segunda, durante la primera mitad del siglo XX; la tercera, a partir de la década de los 60; y la cuarta, a partir de los años 80 hasta nuestros días. Debemos aclarar que estas etapas no están separadas mediante cortes mecanicistas, sino que son heurísticas y en ellas subyacen aspectos de las demás. 


\section{Primera etapa: a fines del siglo XIX y comienzos del XX}

El análisis predominante, en esta etapa, fue la relación directa con la práctica individual del médico y su relación con el paciente, sin vínculo con la situación social o con los bosquejos de la atención institucional. El médico determinaba el tipo de personas que necesitaba para su trabajo. La formación era artesanal, y el personal acudía al trabajo junto al médico en calidad de aprendiz, sin reconocimiento social ni legitimidad académica, hecho que caracterizó “el modelo médico-céntrico" (García, 1972).

\section{Segunda etapa: siglo XX hasta la década de los 50}

Esta etapa tiene dos aspectos: el primero, a partir de la década inicial del siglo XX, cuando nuevos conocimientos científicos y tecnológicos fueron incorporados a la práctica médica. Al mismo tiempo, los hospitales pasaron a tener una nueva orientación en su organización para la atención a los pacientes y se constituían en centros básicos para la formación de los médicos, del personal auxiliar y del personal técnico de la salud, ligados a las necesidades de los servicios, especialmente a los medios complementarios de diagnóstico, tratamiento y rehabilitación (Andrade, 1979). Así surgieron las primeras experiencias para la formación de técnicos de salud, orientados al ejercicio profesional como:

- Enfermeras.

- Laboratoristas - bioanálisis.

- Dental.

- Salud pública.

- Microbiólogo.

- Alimentos.

- Óptico.

- Operador de radiología.

- Documentación médica.

- Bioestadística.

- Rehabilitación y fisioterapia.

El segundo, corresponde a la consolidación de las especialidades médicas, lo cual condujo no sólo a la formación de especialistas, sino también a la formación del personal técnico y auxiliar propio de esas especialidades, e iniciar así la conformación de equipos de trabajo con integrantes profesionales, técnicos y auxiliares.

En estos dos aspectos, los marcos conceptuales para la organización de los planes y programas de estudio, su duración y su legitimación para el ejercicio del trabajo, se establecieron sobre la base de los análisis de los 
requerimientos institucionales de la demanda de atención y los nuevos aspectos considerados en la atención a la salud colectiva.

Los análisis de las políticas de salud, economía y determinantes sociales se mantuvieron al margen, pues predominó el razonamiento organizacional de la gerencia hospitalaria y la incorporación de nuevas tecnologías para la atención a la salud y la consolidación de las distintas especialidades médicas. El modelo de análisis fue "hospital-médico-céntrico" (Foucault, 1978).

\section{Tercera etapa: década de los 60 hasta los 90}

En la década de los sesenta del siglo XX, los gobiernos de las Américas establecieron programas de desarrollo socioeconómico, como procesos armónicos y secuenciales de acción, para ampliar objetivos prioritarios determinados en los planes y programas establecidos para el desarrollo por las Naciones Unidas. La Tercera Reunión Especial de Ministros de la Salud de las Américas reconoció que la salud es una función que se asocia a las políticas generales de los países (Organización Panamericana de la Salud, 1973; Organización Mundial de la Salud, 1971).

Sobre la base de lo expuesto, tanto a nivel mundial como en las Américas, se llegó al reconocimiento de las siguientes dimensiones básicas para el desarrollo de los planes y programas de salud y la formación de los recursos humanos para la salud:

- La salud, como concepto universal, es para cada ser humano un fin y para la sociedad a la que pertenece, un medio. En consecuencia, la salud es un componente fundamental del desarrollo; es el resultado del enlace de esfuerzos que conducen al bienestar social; se reconoce que en la política de los países, es lo que más expresa el crecimiento económico, las reformas institucionales y los cambios en las estructuras de gestión. En esta etapa se consolida el concepto acerca de los problemas de salud como el conjunto de factores que condicionan las enfermedades y su distribución en cada sociedad. Estos factores son del orden biológico, económico, histórico y cultural.

- Una nueva nomenclatura operativa permitió la diferencia de los problemas relacionados directamente con la situación de salud, la cual depende, por otra parte, de la calidad, cantidad y organización de los recursos, métodos y procedimientos para mejorarla. Los primeros se reúnen bajo la denominación de servicios para la salud de las personas, e incluyen atención materno-infantil, nutrición, control de enfermedades transmisibles y crónicas, y todo lo relativo al ambiente. Los segundos respondieron a la denominación de desarrollo de la infraestructura, de los sistemas nacionales de salud y, dentro de ellos, los recursos humanos, profesionales, técnicos y 
auxiliares; los procesos de administración, planificación, evaluación e información; la legislación y la reglamentación; la investigación básica y operativa, y el financiamiento.

- El renacer de la ecología alcanza un mayor significado al reconocerse que la calidad de la vida deriva del continuo intercambio e interrelación de los seres humanos con su ambiente, un verdadero metabolismo social en el que participan todas las variables que es dado suponer, inducidas o producidas por el hombre y la naturaleza. En los años venideros, los gobiernos y las comunidades tendrán que hacer frente a problemas ambientales de mayor magnitud y complejidad. El adelanto de la tecnología ha dejado una estela de tensiones humanas más complicadas; aumentaron los contaminantes ambientales, que abarcan, desde los microbiológicos, hasta aquéllos que proceden de sustancias tóxicas, que adquirieron mayor importancia y serían las de más difícil diagnóstico porque existe una mayor separación entre la causa y el efecto. El crecimiento de las ciudades agravó los problemas de congestionamiento del tránsito, de los accidentes y los riesgos al ruido. Las mayores densidades de población y la vivienda deficiente aumentaron los riesgos de las enfermedades transmisibles, y los problemas de salud mental. En los complejos industriales, las enfermedades profesionales exigieron una atención más concentrada y medidas curativas. En el futuro, los organismos de salud tendrán que ampliar sus actividades con el fin de tomar en consideración los barrios míseros, la pobreza y la suciedad, la ignorancia y la delincuencia, y los efectos que estos factores tienen sobre la salud total de la población.

- La dinámica de la población puso en evidencia que la extensión de los servicios de salud materno-infantiles era limitada, discontinua y de eficiencia restringida. La protección a embarazadas era, generalmente, inferior al $30 \%$; la atención institucional al parto, en cerca de la mitad de los países, era inferior al 50\%; la asistencia en el puerperio con frecuencia estaba por debajo del 5\%. Asimismo, la atención a los menores de cinco años solía ser tardía, de escasa cobertura e inadecuada integración. Los hechos reseñados explican, en gran medida, la debilidad de la infraestructura administrativa de los servicios de salud materno-infantil; la limitación o inexistente asignación de recursos a los programas de referencia; la limitada disponibilidad de personal profesional y auxiliar especializado y su distorsionada distribución; las dificultades existentes en comunicación, transporte y equipamiento, y de un modo especial, la inexistencia de una efectiva participación de la comunidad en los programas correspondientes.

- Alimentación y nutrición generaron un complejo proceso-que se extiende desde la producción y el consumo de alimentos, hasta la utilización biológica-, visualizado como un sistema definido de alimentación y nutrición. Se reconoció que, como parte del desarrollo de una política 
intersectorial de alimentación y nutrición, habían programas que contribuían a la obtención de una solución integral y otras medidas que pueden ayudar a aliviar la situación a corto plazo.

- La participación de la comunidad y sus niveles de organización reconocidos como el elemento decisivo para aprovechar el inagotable potencial de la población, conduciendo sus inquietudes hacia actividades de verdadero servicio social, para el mejoramiento del ambiente. Las repercusiones deben ser trascendentales en la lucha para alcanzar mejores y más altos niveles de salud que propicien el constante desarrollo físico, social, cultural y económico del hombre. La participación de la comunidad fue una respuesta acertada a este nuevo enfoque de atención a la salud y a la enfermedad en cuanto la motivación, la educación y la organización de las comunidades les permitió participar en los programas de salud para su propio beneficio.

En esta etapa, el análisis del contexto político, social y económico pasó a ser la base de la corriente emergente de planificación de la salud y de los recursos humanos con un enfoque heurístico. Este enfoque es válido, tanto para la formación del personal profesional, técnico y auxiliar (en función de llevar adelante el cumplimiento del plan de salud que integre las acciones de promoción, prevención, atención y rehabilitación), como para estructurar los distintos niveles de atención (primaria, secundaria y terciaria), involucrando el estado de salud y enfermedad de toda la población de cada uno de los países, así como sus perspectivas de desarrollo y sus limitaciones para cumplir las metas y objetivos propuestos a mediano y largo plazo.

Este enfoque tuvo una aplicación lenta debido a diversos problemas, entre ellos: las discusiones de planificación de los recursos humanos fuera del contexto de la planificación general de la salud y del plan de desarrollo político, económico y social de los países latinoamericanos; el hacer explícita la separación de la decisión política en salud y educación, principalmente la universitaria, que trajo como consecuencia que por varios años no se hubiera percibido claramente la idea de que la planificación del desarrollo de los recursos humanos no puede tener éxito si no se efectúa como parte integral de todo el proceso de planificación nacional y, en particular, de la salud y la educación; enfoques de planificación, por grupos ocupacionales aislados, según la orientación dada por los servicios. Estos enfoques fueron conceptualmente incorrectos porque el efecto de las actividades de salud en la colectividad no puede ser la acción de un determinado profesional aislado, sin tener en consideración que las funciones que desempeñan unos $u$ otros, son dependientes y complementarios.

Por último, debemos indicar que, además de los problemas que se plantearon, se incorporaron otras limitaciones, como la desaprobación de los aspectos culturales, los costos de la atención de la salud y la sostenibili- 
dad de las inversiones crecientes, en la medida en que se ampliaban los modelos de atención al proceso salud-enfermedad.

De esta etapa, lo más importante fue la incorporación progresiva, en los países de América Latina y el Caribe, de conceptos y metodologías para el establecimiento de una política nacional de recursos humanos que señale la orientación general hacia la cual se debe dirigir el desarrollo de esos recursos en función del cumplimiento de los objetivos políticos, económicos y sociales del país (Testa, 1970; Ministerio de Salud Pública de Colombia -Asociación Colombiana de Facultades de Medicina-, 1969; Organización Panamericana de la Salud, 1973b).

En estas condiciones, las responsabilidades de la planificación, formación y capacitación del personal profesional, técnico y auxiliar para la salud, pasaron del marco conceptual 'hospital-médico-céntrico' a un marco más amplio que podríamos considerarlo como 'socio-institucional-comunidad-céntrico'. Los análisis políticos comprendieron no sólo la relación con los organismos de formación de personal de salud, sino también con la política y el plan que el gobierno estableció para el desarrollo de los distintos sectores (industria, agricultura, etc.). Desde el punto de vista de la educación, involucró lo que sucedía en el campo educacional, con una visión amplia que llevó a considerar armoniosamente la enseñanza primaria, secundaria, técnica y universitaria, y la promoción de la salud según las necesidades del país en diferentes niveles: central, estadual o provincial, y/o local. Además, la política de recursos humanos, a nivel nacional y local, se relacionó con la organización de los servicios de salud según niveles de atención: primario, secundario y terciario, y tomó en consideración, igualmente, los análisis que mostraban la inadecuada distribución geográfica y ocupacional de los recursos humanos. En consecuencia, se hizo evidente que toda medida orientada a mejorar la situación presente, tendría divisiones políticas, en términos del dilema de la responsabilidad social versus libertad individual.

A la dimensión política, se incorporó la evaluación realista del contexto económico del país, incluyendo el comportamiento de costos, el flujo de fondos, las inversiones de capital, la demanda, la competencia, el ingreso fiscal, la oferta y la productividad. De igual importancia se otorgó el análisis de la administración pública, especialmente lo relacionado con la organización gubernamental en el país o a la organización social, su estructura de clases, los factores étnicos, la movilidad social, las características de las comunidades rurales y urbanas, el papel de la familia y la dinámica de los cambios sociales. Se reconoció que un planificador de los procesos de formación del personal de salud debía pensar en términos socioculturales, lo cual facilitaría definir los factores que mantienen los programas públicos de educación y promoción de la salud, control de enfermedades 
transmisibles, ubicación de los servicios, saneamiento ambiental y otras actividades programáticas.

En América Latina, surgió un movimiento importante para la creación de escuelas o politécnicos de la salud para la formación de personal técnico. Aunque manteniendo su relación con las facultades o escuelas de medicina, odontología y enfermería, tendría autonomía relativa para determinar el avance en la formación de este tipo de personal mediante la creación de escuelas y politécnicos de la salud con un perfil educacional amplio y diversificado.

En los países que conformaron el Pacto Andino, a partir de 1970, fueron creadas las primeras escuelas de formación de personal técnico a nivel universitario (Lynch, 1980). En Costa Rica se estableció, para América Central, el Programa de Adiestramiento en Salud Comunitaria (Pascap), que impulsó la preparación de programas para la formación de personal, investigaciones socio-epidemiológicas y material docente-educativo. Además, el Pascap contribuyó a la creación de los institutos tecnológicos en salud de Nicaragua, Honduras y Guatemala (Pascap, 1980). En República Dominicana, fueron creadas dos escuelas para la formación de enfermeras, nutricionistas, educadores para la salud y técnicos en laboratorio clínico (Romero, Silverio y Troncoso, 1980). En Cuba, durante la década de los 80, la Dirección Nacional de Docencia Médica conformó una red de 56 centros educativos con el propósito de formar técnicos medios para la salud; de éstos, 18 eran institutos politécnicos. Para los años noventa, se formaban técnicos a nivel medio para la salud en 28 perfiles de especialidades, con una matrícula de 19.000 estudiantes (Hernández y Márquez, 1976).

Por la importancia que adquirió esta actividad, se realizaron tres talleres internacionales. En el tercero, celebrado en Venezuela, se planteó la organización de un centro latinoamericano de coordinación de instituciones formadores de personal técnico a nivel medio en salud, proyecto que no pudo concretarse. En Brasil, a partir de la década de los 70, surgió un movimiento nacional, federal, estatal y municipal para ampliar la red de servicios de salud y, en consecuencia, la preparación estratégica, a gran escala, de personal auxiliar y técnico, con un modelo propio, coordinado desde el Ministerio de Salud y ejecutado en los estados y municipios (Recursos humanos para las actividades de salud, 1967). Ver Cuadro 1. 


\section{Cuadro 1}

Sistematización de la formación del personal técnico en salud, según área de formación y especializados para la década de los 90

\begin{tabular}{|c|c|}
\hline Área de formación & Especialidades \\
\hline \multirow[t]{6}{*}{ Enfermería } & Enfermería general \\
\hline & Enfermería pediátrica \\
\hline & Enfermería obstétrica \\
\hline & Enfermería naval \\
\hline & Enfermería especial \\
\hline & Anestesiología \\
\hline \multirow[t]{5}{*}{ Laboratorio } & Laboratorio clínico \\
\hline & Banco de sangre y transfusiones \\
\hline & Microbiología \\
\hline & Químico sanitário \\
\hline & Gastroenterología \\
\hline \multirow[t]{4}{*}{ Radiología } & Radiología clínica \\
\hline & Radioterapia \\
\hline & Medicina nuclear \\
\hline & Proteccíon contra radiaciones \\
\hline \multirow[t]{2}{*}{ Salud mental } & Psicometría \\
\hline & Trabajador social de la salud \\
\hline \multirow[t]{3}{*}{ Patología } & Anatomía patológica \\
\hline & Citotecnología \\
\hline & Patología médico-legal \\
\hline \multirow[t]{5}{*}{ Rehabilitación } & Fisioterapia \\
\hline & Ergoterapia \\
\hline & Mecánico ortopédico \\
\hline & Ortopedia \\
\hline & Pediatría \\
\hline \multirow[t]{3}{*}{ Salud pública } & Trabajador sanitario \\
\hline & Estadística de salud \\
\hline & Dietista de salud \\
\hline \multirow[t]{2}{*}{ Bibliotecología } & Bibliotecaria médica \\
\hline & Iconofotografía \\
\hline \multirow[t]{3}{*}{ Órganos de los sentidos } & Oftalmología \\
\hline & Logopedia y foniatría \\
\hline & Audiología \\
\hline \multirow[t]{2}{*}{ Farmacia } & Farmacéutico \\
\hline & Industria farmacéutica \\
\hline \multirow[t]{3}{*}{ Estomatología } & Estomatología clínica \\
\hline & Prótesis dental \\
\hline & Asistente dental \\
\hline \multirow[t]{2}{*}{ Electromedicina } & Electrónica médica \\
\hline & Equipo de rayos $\mathrm{X}$ \\
\hline
\end{tabular}




\section{Cuarta etapa}

A finales de los años 70 del siglo XX, América Latina y los países en desarrollo tuvieron que soportar una profunda crisis económica e inflacionaria, determinada por el sesgo capitalista que adoptaron los gobiernos de ese período, con consecuencias nefastas para la población, que década tras década soportó un desarrollo desigual e inequitativo que elevó al $41 \%$ los niveles de pobreza, al $275 \%$ la inflación, aumento la deuda externa a niveles impagables, provocó la pérdida de miles de puestos de trabajo, redujo el nivel educacional y de la atención a la salud, y profundizó las desigualdades sociales y económicas (Banco Mundial, 1993).

En ese momento, dos organismos supranacionales, el Fondo Monetario Internacional y el Banco Mundial, asumieron la defensa de los acreedores, preconizando e imponiendo políticas de ajuste estructural que determinaron el control económico-social de los países latinoamericanos. Estas políticas operaron y operan en la dirección de ajustar o contener los gastos.

Bajo el impulso de esas dos instituciones, las acciones de salud pública se redujeron a un conjunto muy limitado de medidas y servicios clínicos esenciales, con medicamentos de bajo costo. Se enfatizó en las campañas contra el tabaquismo, las bebidas alcohólicas y otras substancias aditivas, mientras que las acciones de mayor complejidad fueron delegadas a proveedores eficientes y responsables, identificados con el sector privado. Se limitó el servicio a cinco áreas de trabajo:

- Servicios para garantizar asistencia en el embarazo, parto y puerperio.

- Servicios de planificación familiar.

- Lucha contra la tuberculosis.

- Lucha contra las enfermedades de transmisión sexual.

- Tratamiento de enfermedades comunes de los niños hasta los cinco años (diarreas, infecciones agudas respiratorias, sarampión, paludismo y mala nutrición grave).

Para la atención de la gran mayoría de los problemas del complejo proceso salud-enfermedad, se implementó la norma del Banco Mundial: la atención de salud pasaría a los planes de seguro, autofinanciados por la comunidad, lo cual liberaría los fondos estatales para el ahorro nacional con el fin de que los países pudieran cumplir con los pagos correspondientes a los servicios e intereses de la deuda externa.

Con relación a lo anterior, dos aspectos se destacan de esta etapa, fueron la orientación para subsidiar la práctica privada y la privatización de los servicios públicos, con el propósito de promover la diversidad y la competencia; y la conformación de seguros de salud para que el pago anticipado garantizara, junto a los subsidios del estado al sector privado, la recau- 
dación de los costos y el autofinanciamiento (Organización Mundial de la Salud, 2000).

La OMS se unió a este modelo. En su informe del año 2000, planteó la ruptura de los paradigmas de los sistemas de salud que se conformaron durante las cuatro últimas décadas del siglo XX, particularmente, desde la Reunión de Alma-Atá en 1978 y la estrategia de Salud para Todos. En la actualidad, muchas de las reformas llamadas de tercera generación, parten de la idea de responder a la demanda que a las necesidades de la población; que se manifiesta a los cambios políticos y económicos de la era de la globalización neoliberal y que corresponden al abandono de la planificación del desarrollo, como tarea central del Estado, para dar paso a la planificación que se basa en las leyes del mercado, y asignar mayor importancia a la capacidad, decisión y responsabilidad del sector privado. Esto ha incrementado el interés por los mecanismos de los seguros privados. En el Gráfico 1, tomado del Informe de la OMS, se expone las características de organización de un sistema de salud que contempla el control administrativo, que se centraliza en las leyes del mercado.

\section{Gráfico 1}

Características de organización de un sistema de salud que contempla el control administrativo

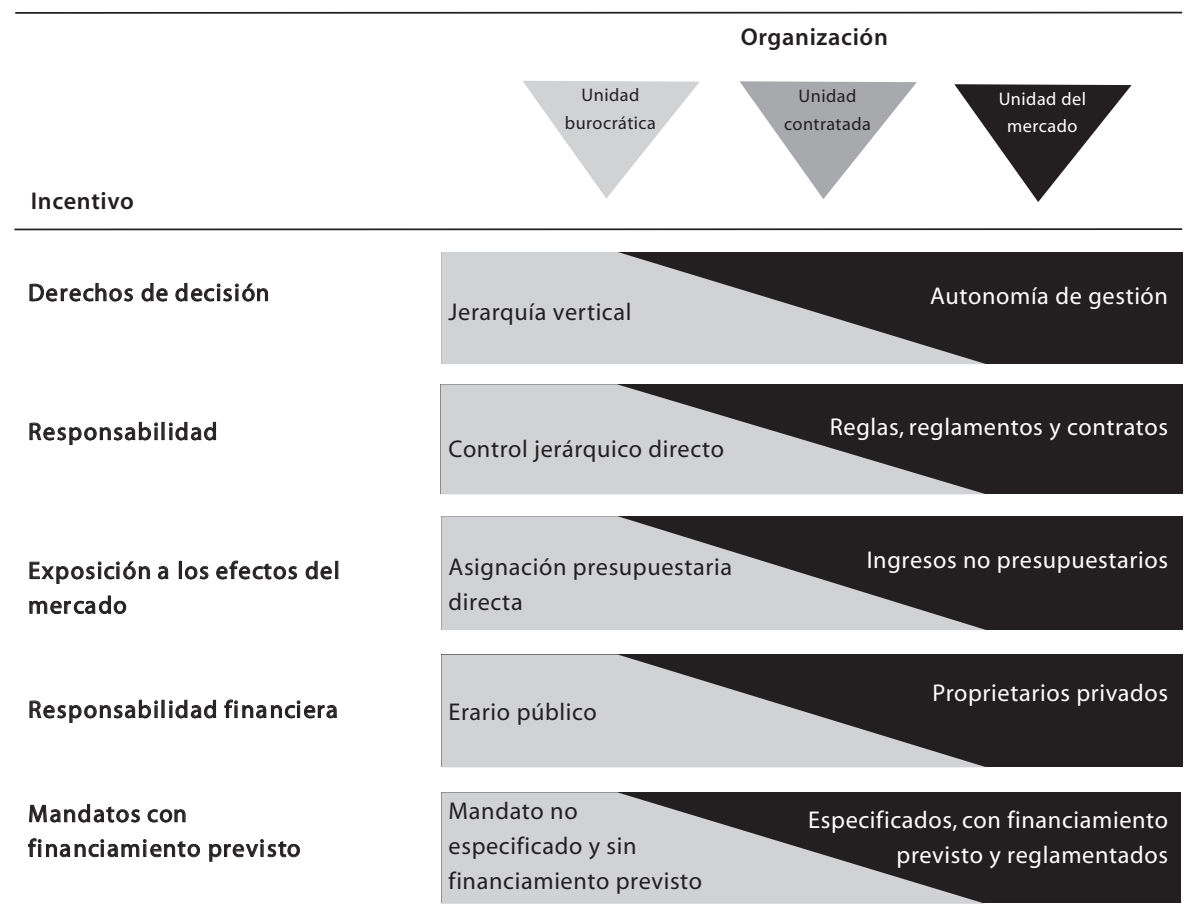


La introducción del mercado, progresivamente, en el discurso y en la práctica de los sistemas de salud, transformó al paciente en un cliente y a la planificación en salud en mercados sanitarios.

En el informe de la OMS del año 2000, el modelo colombiano es considerado como el ideal para los países latinoamericanos, por el hecho de haber reemplazado su sistema de salud, su estructura institucional en los distintos niveles de atención, por un sistema de protección privada; en cambio, considera al modelo cubano como anacrónico y, sin detenerse a analizar objetivamente lo que pasó con las condiciones de salud de la población de Colombia y de Cuba, respectivamente, pretendía el traslado del modelo colombiano, acríticamente, a otros países, ocultando su propia realidad.

El panorama, expuesto en forma concisa, tuvo diferentes respuestas en otros países: Cuba continúa profundizando la aplicación de su tesis central, según la cual la salud es un derecho indispensable de todas las personas y una responsabilidad del Estado, acompañado de la activa participación de la población como sujeto y objeto de la atención de la salud; Venezuela construye su propio sistema de salud fuera del marco de las organizaciones supranacionales; Bolivia emerge con un modelo propio, basado en su gran acerbo cultural e histórico, de profundas raíces quechuas y aimaras; Argentina reforma sus planes como Nación, como razón de ser de las políticas económicas y sociales; Uruguay refuerza su sistema nacional de salud; Brasil avanza con relación a los planteamientos del Movimiento Sanitarista de mediados de la década de los 70 del siglo $\mathrm{XX}$, y toma los distintos aspectos de la atención a la salud del pueblo brasileño como una conquista colectiva para disminuir las profundas desigualdades existentes en la sociedad y mantener, como un derecho constitucional y un deber del Estado, la atención universal a la salud del pueblo, reafirmando el principio de la atención medico-sanitarista integral, de carácter universal, con acceso igual para todos, con participación ciudadana y una ejecución descentralizada de los planes y programas sanitarios.

En el complejo contexto internacional y nacional, el marco conceptual para la formación del personal de la salud pasó a tener una proyección que se basa en las posiciones extra-nacionales, homogeneización, acríticas y antehistóricas que, en vez de ampliar las líneas de análisis, optaron por un reduccionismo empirista de las llamadas reformas del sector salud de primera, segunda y tercera generación. Estas reformas siguen el modelo neoliberal y se refieren, fundamentalmente, a la relación de las empresas de atención de salud con las leyes desreguladas del mercado; aplican el principio de competencias individuales con la eliminación de los contratos de personal, de permanencia en el trabajo con derechos adquiridos y acumulables para el término de la vida laboral, y dan paso a una concepción de flexibilidad tecno-organizativa y la introducción de tecnologías informatizadas para deter- 
minar las condiciones de trabajo, remuneración y los tiempos de contratación, que llevan, en último término, a aumentar las tasas de ganancias y capitalización empresarial.

En esta disyuntiva, se planteó la transformación de la gestión de la fuerza de trabajo, privilegiando - en la definición de perfiles y requisitos para la formación y capacitación del personal- nuevas variables, tales como el aporte formador en el que prime el eficientísimo, el desempeño individual y la capacidad para adaptarse al mercado laboral dominado por el pacto empresario-trabajador; la competencia y la constante rotación en su trabajo, según las demandas del mercado, sin interés por lo que pasa en toda la sociedad o por las necesidades que siente la población; cada vez más sometidos a los métodos tecnológicos, de la eficiencia y efectividad en el trabajo que posibilite la acumulación de capital de la empresa de salud, según un modelo preestablecido. La siguiente figura ofrece una síntesis de los cambios propugnados para configurar el marco de referencia para los análisis que deben prevalecer en el establecimiento del marco conceptual para la formación del personal de la salud.

\begin{tabular}{|c|c|}
\hline \multicolumn{2}{|c|}{ Reformas sectoriales y gestión de recursos humanos } \\
\hline \multicolumn{2}{|c|}{ Reformas económicas y estatales } \\
\hline $\begin{array}{l}\text { - Cambios en los sistemas e ocupación } \\
\text { funcionaria } \\
\text { - Énfasis en la profesionalización } \\
\text { - Cambios del régimen laboral y salarial. } \\
\text { Precarización } \\
\text { - Cambios en la regulación de mercados } \\
\text { de trabajo }\end{array}$ & $\begin{array}{l}\text { - Disminución de recursos públicos } \\
\text { - Reducción del personal } \\
\text { - Flexibilidad laboral } \\
\text { - Énfasis en la evalución del desempeño } \\
\text { y capacitación del personal }\end{array}$ \\
\hline \multicolumn{2}{|c|}{ Gestión descentralizada de RRHH de Salud } \\
\hline \multicolumn{2}{|l|}{ Reforma sectorial } \\
\hline \multicolumn{2}{|l|}{ Descentralización } \\
\hline \multicolumn{2}{|l|}{ Cambios en el papel de los ministerios } \\
\hline \multicolumn{2}{|l|}{ Variaciones en el financiamiento de servicios } \\
\hline \multicolumn{2}{|l|}{ Cambios en la organización y gestión } \\
\hline \multicolumn{2}{|c|}{ Énfasis: en la recuperación de costos y la eficiencia } \\
\hline \multicolumn{2}{|c|}{ Garantía de la calidad: habilitación, categorización y acreditación } \\
\hline Sistemas de incentivos & \\
\hline
\end{tabular}


El incremento de la autonomía de la gestión de los empresarios en los distintos niveles de atención directa e individual del cliente, en el control tecnológico y, sobre todo, en la definición de los costos, utilización de los medicamentos, es el elemento central para completar la fase acumulativa del capital de las empresas nacionales o transnacionales.

En el modelo utilizado para el enfoque, se propugnó los siguientes aspectos:

- Eliminación, en los modelos de atención, de los sistemas de carrera de funcionario de servicio público para dar paso a sistemas que se basan en la evaluación del desempeño individual.

- Énfasis en los procesos formativos, teniendo como base la competencia en los mercados laborales.

- Orientación de los regímenes laborales, enfatizando la flexibilidad en las fluctuaciones de los mercados de trabajo.

- Generación de nuevas formas en los regímenes salariales, enfatizando los incentivos por productividad y desempeño.

- Desregulación de los mercados laborales.

- Disminución masiva del personal institucional mediante despidos masi-vos, eliminación de puestos de trabajo, congelación de puestos o compra de renuncias.

- Limitación del tiempo de los contratos, eliminación de la carrera profesional e incremento de la modalidad de los contratos a tiempo limitado o por servicios presentados.

Desde esta óptica, surgieron nuevos marcos de referencia para el desarrollo del personal de la salud, caracterizados por el énfasis en la descentralización de la gestión de servicios, con un cambio en el papel del Estado, que pasa a actuar como regulador de las relaciones empresa-cliente y se convierte, además, en un contribuyente más al financiamiento de los servicios de salud a nivel secundario y terciario, que ofrece el sector privado según la oferta y la demanda, y el incremento de los costos.

La otra corriente, entre tanto, mantuvo su enfoque institucional: el análisis del contexto político, económico y social para conformar el marco conceptual de la formación del personal de salud, construido durante décadas para lograr la atención en salud equitativa, universal, de acuerdo con el desarrollo integral del país, en que el componente de salud es fundamental para el desarrollo del capital humano, cada vez más capacitado y comprometido con la sociedad para lograr el bienestar ciudadano. En esta etapa, si bien se mantuvo el modelo determinado para la tercera etapa, se incorporaron nuevas dimensiones en los análisis contextuales, tales como:

- Cómo interpretar las políticas globales en salud en el mundo, sus contradicciones, limitaciones y la sistematización de experiencias ocurridas en el siglo XXI en las diferentes corrientes. 
- La explicitación de la problemática en el campo de la formación de recursos humanos para la salud, de lo cual resultan los siguientes aspectos: desequilibrios estructurales de la fuerza de trabajo y la mala distribución (en relación con diversos criterios); sueldos públicos y poco competitivos, y heteronimia entre subsectores, como, por ejemplo, entre ministerios y seguridad social; heterogeneidad e inadecuación de regímenes laborales con tendencia creciente a la desinstitucionalización; insuficiencia de criterios e instrumentos de evaluación; conflictividad laboral larvada o evidente, que llega a interferir con el proceso de producción de servicios; fragmentación, heterogeneidad y complejidad creciente de los procesos de incorporación y utilización tecnológica; aumento de los costos de trabajo sin capacidad de control de la gestión existente; asumir los retos que se generan en los cambios ocurridos con el paso a una gestión descentralizada, con reconocimiento de la importancia de las unidades básicas de la sociedad, como los municipios. La alternativa planteada se fundamenta en el reconocimiento de la importancia del cambio de una gestión normativa a la estratégica; del verticalismo a la participativa; de los objetivos de orden y control a objetivos de productividad y calidad; de una visión fragmentada a una visión integrada.

- Mirar, hacia otra perspectiva, la organización del trabajo sobre la base de la información, que permita: promover el desarrollo de sistemas de información para adoptar decisiones que eleven la productividad y la calidad; incorporar el análisis del desempeño de los equipos de salud; redefinir las pautas de desempeño de los equipos de salud; redefinir y asignar el personal necesario; promover la evaluación permanente de procesos y resultados; promover el trabajo en equipo y estimular la participación; desarrollar la investigación operacional necesaria.

En esta etapa, resultó necesaria la participación de las unidades nacionales de ciencia y tecnología en la conformación de las redes nacionales o internacionales para el acceso, utilización racional y discriminada de las tecnologías y productos, sin plegarse al pensamiento que se genera bajo los principios de la globalización y las leyes del mercado que, indudablemente, privilegian los enfoques científicos transnacionales. Según estos enfoques, los países periféricos son meros receptores pasivos de los resultados, y están limitados para conformar sus propios sistemas de ciencia, tecnología e innovación que respondan a necesidades propias y permitan alcanzar nuevos niveles en las capacidades humanas y una vida más larga y saludable de su población. En los análisis de esta dimensión, en el campo de la salud, resulta de gran importancia conocer, analizar y adoptar posiciones ante las profundas transformaciones del sector farmacéutico, influenciado por la tendencia a universalizar la mercantilización de los medicamentos, según los valores del mercado, la libre elección y la gestión privada. 
- El marco conceptual para la formación de personal de la salud se perfecciona con la salud ambiental y ecológica, y se reconocerán los conocimientos sobre el medio ambiente, aún no se han organizado junto a una doctrina científica unificada.

\section{Nota}

1 Médico sanitarista, professor convidado da Universidade de Havana e honorário da Universidade de Cuenca. Membro da Organização Pan-Americana da Saúde (Opas).

\section{Referências}

ANDRADE, J. Marco conceptual de la educación médica en la América Latina. Washington, D.C. Organización Panamericana de la Salud. Serie Desarrollo en Recursos Humanos, n. 28, 1979.

BANCO MUNDIAL. Informe sobre el desarrollo mundial. Invertir en salud. Madrid, Mundi Prensa S. A., 1993.

FOUCAULT, M. Medicina e Historia: El pensamiento de Michel Foucault. Washington, D.C. Organización Panamericana de la Salud, 1978.

GARCÍA, J. C. La educación médica en América Latina. Washington D.C. Organización Panamericana de la Salud. Publicación Científica n. 225, 1972.

HERNÁNDEZ, E.; MÁRQUEZ, M. Docencia Médica Media. Educación Médica y Salud, n. 1, v. 10, 1976.

LYNCH, A. Actividades de recursos humanos en el Pacto Andino. Venezuela, II Taller Latinoamericano sobre la Formación de Técnicos Medios en Salud. Informe Final, 1980. Documento mecanografiado.

MINISTERIO DE SALUD PÚBLICA. Asociación Colombiana de Facultades de Medicina. Colombia. Estudio de recursos humanos para la salud y la educación médica en Colombia. Bogotá: Ascofame, 1969.

ORGANIZACIÓN MUNDIAL DE LA SALUD. Estudios sobre personal de salud. Informe de un grupo científico de la OMS, n. 481, 1971.

Informe sobre la salud en el mundo. Mejorar el desempeño de los sistemas de salud. Ginebra: OMS, 2000.

ORGANIZACIÓN PANAMERICANA DE LA SALUD. Plan Decenal de las Américas. Informe Final de la III Reunión Especial de Ministros de las Américas. Washington, D.C. OPS/OMS. Documento Oficial 118. $1973 a$.

Problemas y perspectivas de la planificación de recursos humanos para 
la salud en América Latina (Conferencia Panamericana sobre Planificación de Recursos Humanos en Salud). 1973b. Educación Médica y Salud, v. 7, n. 3-4, 1973.

PASCAP. Programa de adiestramiento en salud comunitaria para la atención primaria en Centroamérica y Panamá. Comacú, Venezuela. II Taller Latinoamericano sobre Formación de Técnicos Medios en Salud. Informe Final, 1980.

RECURSOS HUMANOS PARA AS ATIVIDADES DE SAÚDE. Análisis de la IV Conferencia Nacional de Salud, 1967, Rio de Janeiro, Brasil, Fensp.
ROMERO, M.; SILVERIO, R.; TRONCOSO, M.C. La formación de personal técnico medio en salud. Venezuela, II Taller Latinoamericano sobre Formación de Técnicos Medios en Salud. Informe Final, 1980.

TESTA, M. Planificación de recursos humanos para la salud con respecto a tipos, cantidad y adecuación a la función. Educación Médica y Salud, v. 4, n. 1-2, 1970.

Recebido em 29/08/2008

Aprovado em 23/10/2008 
